\title{
The ability to orient attention in Gilles de la Tourette syndrome
}

\author{
Debra Howells*, Nellie Georgiou-Karistianis \\ and John Bradshaw \\ Neuropsychology Research Unit, Psychology \\ Department, Monash University, Clayton 3168, \\ Victoria, Australia
}

\begin{abstract}
Gilles de la Tourette syndrome is characterised by tics, although patients also commonly present with attentional problems. This experiment aimed to ascertain whether TS patients have problems in orienting attention, via the use of a vibrotactile choice reaction time task. Participants were required to push a button in response to a faint vibration delivered to the index finger. Prior to each stimulus vibration, a pre-cue (valid, neutral or invalid) was administered to the finger. The performance benefits and costs participants gained from valid and invalid precues were calculated. Contrary to our prediction TS patients did not show significantly different costs or benefits. Furthermore, both patients and controls showed an equal and increased benefit in the crossed arm posture, compared to the uncrossed. These results suggest TS patients do not generally have problems in orienting attention. In addition, in the tactile modality, both patients and controls may benefit from directed attention when difficulty levels are maximal.
\end{abstract}

Keywords: Tourette's syndrome, attention, orienting, cues, costs, benefits

\section{Introduction}

Gilles de la Tourette syndrome (TS), a neurodevelopmental disorder, is characterised by motor and vocal tics. The average age of onset is seven years, affecting three times more males than females [13]. Although its aetiology is unknown, debate continues regarding the mode of inheritance $[5,6,7]$. Brain imaging studies consistently implicate fronto-striatal pathways, with a unilateral volume reduction in the putamen and globus pallidus [17, 23], as well as an imbalance of dopamine in the basal ganglia and limbic system [16].

Attentional impairments have been well documented in TS patients; however the nature of these

\footnotetext{
* Corresponding author: Debra Howells, Neuropsychology Research Unit, Psychology Department, Monash University, Clayton 3168, Victoria, Australia. Tel.: +61 39905 3933; Fax: +61 39905 3948; E-mail: debra.howells@sci.monash.edu.au.
}

problems is unclear. Furthermore, much of the research has focused on children and consequently there is limited research investigating attentional problems in adult TS patients. Channon et al. (1992) [4] reported adult TS patients were disadvantaged on Trail Making Test B and a letter cancellation task, suggesting problems in holding attention. To further elucidate the nature of attention impairments, Georgiou et al. (1995) [8] investigated the ability of TS adults to shift attention from spatially congruent and incongruent visual stimuli, using a Simon effect paradigm. TS patients had difficulty responding to conflicting stimulusresponse configurations, due perhaps to problems in making attentional shifts and/or inhibition of inappropriate responses.

In a further study employing a vibro-tactile choice reaction time task, Georgiou et al. (1996) [9] investigated the ability of adult TS patients to hold their attention at expected locations and to shift their attention to unexpected locations. The authors reported TS patients were overall slower to respond than controls, but were not very much more disadvantaged at shifting or holding attention. Using the same vibro-tactile procedure, Georgiou et al. (1998) [10] investigated the effect of gaze on the ability of adult TS patients to hold and shift attention. Patients were required to either 'look at' or 'away' from the stimulus hand. Although TS patients did not benefit from directed attention (gaze), patients failed to show normal expectancy effects, suggesting more difficulties in holding than in shifting attention.

In attempt to further elucidate the nature of attentional impairments in adult TS patients we employed a Posner-type cost-benefit paradigm [18], using valid, neutral and invalid precues, in a vibro-tactile choice reaction time task. This paradigm permits a costbenefit analysis from invalid and valid cues respectively, together with dissociation of the effects of disengaging, shifting and holding of attention. Rafal et al. (1988) [21] explain that the efficiency of moving attention can be inferred from performance facilitation (benefits) on valid precue trials, and problems in disengaging attention can be inferred from the costs associ- 
ated with invalid precues (i.e., attention is disengaged from the cue before reorienting to the new target). Furthermore, as one would expect faster reaction time's (RT) on valid trials, similar RT performance on valid and invalid trials may suggest problems in holding attention. Therefore, we employed this paradigm in attempt to further understand the nature of attentional impairments in adult TS patients.

\section{Methods}

\subsection{Participants}

Twelve TS and 12 controls participated, ten males and two females, all of which were right handed. Controls were matched to TS participants by sex, age (within two years) and IQ. Patients, all volunteers from the Victorian Tourette Syndrome Association, met DSM-IV [1] diagnostic criteria. Symptom severity was assessed using the Tourette's syndrome Global Scale (TSGS) [11], with a mean score of 25.12. Five patients had either co-morbid OCD, ADHD, or both and the remaining seven had pure TS. Two TS patients were unmedicated, while the remaining ten were on either haloperidol, methylphenidate, pimozide, prozac, lithium, diazepam, or fluoxamine. It has been previously demonstrated that medication status of TS patients (i.e., whether on or off) has no influence on attention performance [8].

All participants were screened for dementia using the Short Test of Mental Status (STMS) [12]. TS scores did not differ significantly from controls', $(F(1,22)=1.29, P>0.27)$. Furthermore, each participant was assessed for depression using the Mood Assessment Scale (MAS) [24]. TS participants were significantly more depressed than controls, $(F(1,22)=8.02, P<0.01)$. Finally, participants' full I.Q. was predicted using the National Adult Reading Test (NART) [15]. There were no significant differences between TS and control participants' scores, $(F(1,22)=0.522, P>0.48)$.

\subsection{Apparatus}

Two Oticon-A (47 $\Omega$ impedance) bone conductors acted as transducers, with vibrating surfaces of $1.7 \mathrm{~cm}$ in diameter. They were driven by oscillators under the control of a Toshiba portable computer. The vibrotactile stimuli were set at a frequency $(250 \mathrm{~Hz})$, intensity (6 $\mathrm{V}$ peak-to-peak), duration $(80 \mathrm{msec})$ and rise and fall times $(20 \mathrm{msec})$ that produced a clear discernible signal. Participants pressed one of two buttons in response to the vibrotactile stimulus.

\subsection{Procedure}

Subjects sat with arms extended, and the index finger of each hand resting on a button. Vibrotactile transducers were attached with Velcro to the topside of each index finger, leaving the pad free to respond. Subjects fixated straight ahead, with arms either crossed or uncrossed, and responded as quickly as possible by depressing the button on which the stimulated finger rested.

Trials, totaling 360, were presented in blocks of twelve. In each block, six trials were directed to the left hand and six to right, in a random order. A precue of $200 \mathrm{msec}$ duration was presented prior to each stimulus, with an interstimulus interval of $300 \mathrm{msec}$. Precues were either valid, neutral or invalid. Valid precues involved precue and stimulus being delivered to the same finger, whereas invalid cues involved precue and stimulus being delivered to opposite fingers. The ratio, and number, of valid, invalid and neutral trials is most important in designing a Posner-type experiment; for a cue to be useful and informative, the number of valid trials must obviously exceed invalid, and the minimum ratio of $75: 25$ was adopted here, with a possibly-impaired population, instead of the more usual $80: 20$ ratio [3]. Therefore, in this study, 20 out of 30 blocks consisted of valid and invalid cues, in a ratio of $75: 25$. The remaining 10 blocks consisted of neutral cues, where both hands were precued simultaneously. Blocks were arranged such that every third block consisted of neutral precues, and the configuration of each block was alternatively crossed and uncrossed. The ratio of neutral cue blocks to valid/invalid blocks $(10: 20)$ reflects the three-way distribution of trials (valid, invalid, neutral). This yields the figure of 180 valid, 60 invalid and 120 neutral. In total there were 12 conditions corresponding to all possible combinations of configuration (crossed and uncrossed) and precue type (valid, neutral and invalid). The order of block presentation was counterbalanced across subjects.

Initially, 36 practice trials were performed to overcome practice effects and to stabilise RT. Errors were eliminated from analysis. Furthermore, anticipatory RTs below $150 \mathrm{msec}$ were disregarded, as were those above $1000 \mathrm{msec}$. Moreover, RTs more than 3 standard deviations above the subject's overall mean were 
replaced by this value, in accordance with standard procedures [2].

\subsection{Data Analysis}

A random selection of 3 valid and 3 neutral cue trials were preselected from their respective blocks, to equalise the number of trials consisting of valid, invalid and neutral cues.

\section{Results}

Data were submitted to a four-way ANOVA, with factors Group (TS, controls), Configuration (uncrossed, crossed), Cue (valid, neutral, invalid), and Side (side of stimulus - left, right), with repeated measures on the last three factors. Although there were no main effects or interactions involving Group or Side, there was a highly significant main effect of Configuration, $(F(1,22)=49.82, P<0.001)$, with uncrossed (472 msec) $46 \mathrm{msec}$ faster than crossed $(518 \mathrm{msec})$. There was also a highly significant main effect of Cue, $(F(2,44)=44.40, P<0.001)$, with valid cues (456 msec) $50 \mathrm{msec}$ faster than neutral (505 msec), and neutral cues $18 \mathrm{msec}$ faster than invalid $(524 \mathrm{msec})$. There was also an interaction of Cue by Configuration, $(F(2,44)=24.18, P<0.001)$, see Fig. 1 .

One-way ANOVAs involving Cue (valid, neutral, invalid) were then performed separately, for

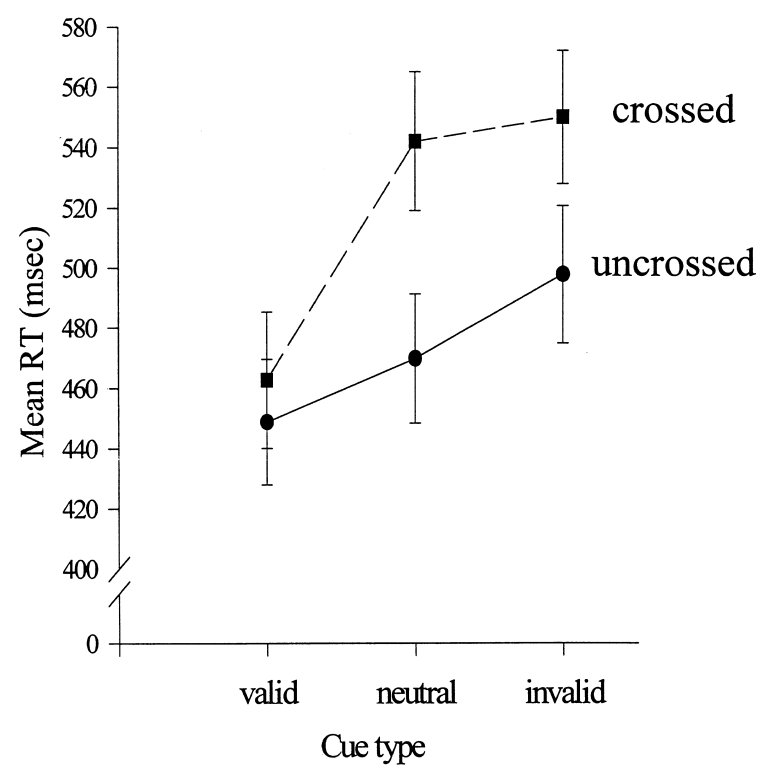

Fig. 1. Mean reaction time (msec) as a function of Cue type (valid, neutral, invalid) for uncrossed and crossed configurations. SE bars included. crossed and uncrossed arm postures. Significance was achieved for both crossed, $(F(2,46)=56.37, P<$ $0.001)$ and uncrossed, $(F(2,46)=19.28, P<0.001)$ postures. Further post-hoc one-way ANOVAs revealed that in the uncrossed posture valid trials were significantly faster than neutral, $(F(1,23)=$ $7.84, P=0.01)$, and neutral faster than invalid trials, $(F(1,23)=12.55, P<0.05)$. Similarly, in the crossed posture, valid trials were faster than neutral, $(F(1,23)=95.62, P<0.001)$; however, no difference was observed between neutral and invalid trials.

Benefits and costs were calculated for TS patients and controls. Benefits were calculated by comparing the speed of responding to valid and neutral cues, whereas costs were calculated by comparing the speed of responding to invalid and neutral cues. Data for benefits and costs were separately submitted to 2-way ANOVA's, with factors Group (TS, control), and Configuration (uncrossed, crossed). A significant effect of Configuration was found for benefits, such that there was a significantly greater benefit in the crossed arm posture, $(F(1,46)=43.02, P<0.001)$, see Fig. 2 a. Similarly, significance was achieved for costs, with greater costs obtained in the uncrossed arm posture, $(F(1,46)=4.92, P<0.05)$, see Fig. 2 b.

The overall error rate was greater in the crossed arm posture $(4.8 \%)$ compared to the uncrossed posture $(1.3 \%)$. Furthermore, more errors were made in the invalid $(11.3 \%)$ and neutral $(11.9 \%)$ trials compared to the valid $(4.1 \%)$ trials, in the crossed posture. Similarly, more errors were made in the invalid (5.8\%) trials compared to the neutral $(1.6 \%)$ and valid $(0.5 \%)$ trials in the uncrossed arm posture. The overall error rate was $2.7 \%$ for TS patients and $3.2 \%$ for controls.

To determine whether performance on the STMS, MAS, NART and TSGS affected overall RT performance for each of the groups, a series of correlations were conducted. Separate analysis of TS and control data revealed significant negative correlations between RT and STMS, $(r=-0.68, P<0.015)$ and $(r=-0.69, P<0.014)$ respectively. No other correlations reached significance.

\section{Discussion}

This study employed a Posner-type cost-benefit paradigm to investigate TS patients' ability to orient attention in the tactile modality. The benefits and costs associated with valid and invalid precues respectively were equivalent for both patients and controls. Fur- 
(a) Benefits

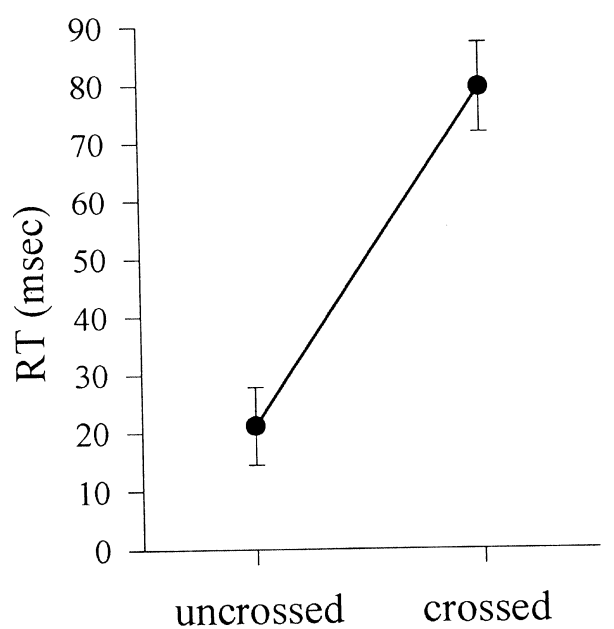

(b) Costs

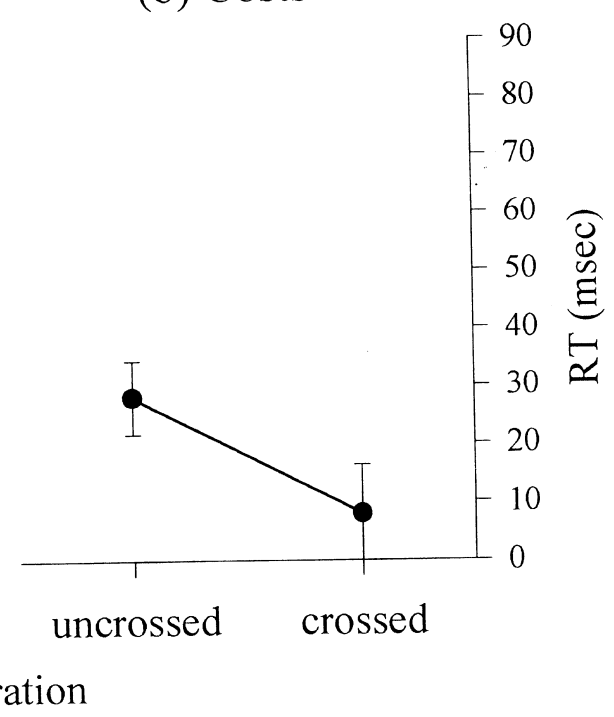

Fig. 2. (a) Benefits (msec) as a function of Configuration (uncrossed, crossed). SE bars included. (b) Costs (msec) as a function of Configuration (uncrossed, crossed). SE bars included.

thermore, for both groups, overall performance was faster in the uncrossed condition, compared to the crossed condition, due probably to a conflict between the anatomical location of the hand and its spatial location while in the crossed position [14, 22]. Overall data showed a valid cue superiority for both groups.

The act of orienting attention can be conceptualised in terms of three operations; disengagement from a current location, shifting attention to a new target location, and finally engaging/holding attention at the new target [19]; these can be operationalized using the present paradigm. While waiting for the precue attention is not engaged. However, when the precue is presented attention must move and engage at the cued location, in anticipation of the target. Thus, the benefit gained from directing attention, using valid precues, reflects the efficiency of moving attention. If however, the target does not occur at the precued location then attention must disengage from the previous cued location before moving to the new target location. Thus, a problem in the operation of disengaging attention is reflected in increased costs associated with invalid precues [21]. Problems in holding attention are thought to reflect similar RT's for both valid and invalid precue trials [20]. Since both groups performed faster with valid than invalid trials, irrespective of arm posture, there was no evidence of impairments in holding attention.

The present findings suggest that adult TS patients do not generally have problems in orienting attention in the tactile modality; however, when coding difficulty levels were increased by forcing the adoption of a crossed arm posture, both the controls and patients showed an equal and increased benefit, compared to the uncrossed arm posture. Thus, when difficulty levels increased both groups equally and more efficiently utilised the external valid information, compared to when difficulty levels were minimal. In contrast, the costs associated with invalid precues were greater in the uncrossed arm posture compared to the more complex crossed posture. The high error rate in the crossed invalid and neutral conditions, together with the reduced costs in the crossed configuration, suggests a possible trade-off between accuracy and cue effect in the more complex posture. Hence, greater costs in the uncrossed arm posture.

Contrary to previous work $[4,10]$, these results suggest that TS patients do not have problems in orienting attention towards a target location in the tactile modality. We suggest that both patients and controls may benefit from the provision of directed attention in tactile tasks when difficulty levels are maximal.

\section{Acknowledgements}

We would like to thank the Victorian Tourette Syndrome Association of Victoria for all their help and cooperation, and all of the members who participated. Sincere thanks goes to Bob Wood, Frank Devlin, 
Truong Nguyen, Andrew Lao and Mike Durham. This work was supported by grants from the Australian Brain Foundation and Australian Research Council.

\section{References}

[1] American Psychiatric Association, Diagnostic and Statistical Manual of Mental Disorders, 4th ed., Washington, DC: Author, 1994.

[2] J. Bradshaw, M. Howard, J. Pierson, J. Phillips and J. Bradshaw, Effects of expectancy and attention in vibrotactile choice reaction time tasks, The Quarterly Journal of Experimental Psychology 44A(3) (1992), 509-528.

[3] J. Bradshaw, M. Waterfall, J. Phillips, R. Iansek, J. Mattingley and J. Bradshaw, Re-orientation of attention in Parkinson's disease: An extension to the vibrotactile modality, Neuropsychologia 31(1) (1993), 51-66.

[4] S. Channon, D. Flynn and M. Robertson, Attentional deficits in Gilles de la Tourette syndrome, Neuropsychiatry, Neuropsychology and Behavioral Neurology 5(3) (1992), 170177.

[5] D. Comings, Tourette's Syndrome: A behavioral spectrum disorder, in: Behavioural neurology of movement disorders: Advances in neurology, Vol. 65, W.J. Weiner and A.E. Lang, eds, Raven Press Ltd., New York, 1995, pp. 293-303.

[6] D. Curtis, M. Robertson and H. Gurling, Autosomal dominant gene transmission in a large kindred with Gilles de la Tourette syndrome, British Journal of Psychiatry 160 (1992), 845849.

[7] V. Eapen, D.L. Pauls and M.M. Robertson, Evidence for autosomal dominant transmission in Tourette's syndrome: United Kingdom cohort study, British Journal of Psychiatry 162 (1993), 593-596

[8] N. Georgiou, J. Bradshaw, J. Phillips, J. Bradshaw and E. Chiu, The Simon effect and attention deficits in Gilles de la Tourette's syndrome and Huntington's disease, Brain 118 (1995), 1305-1318.

[9] N. Georgiou, J. Bradshaw, J. Phillips and E. Chiu, The effect of Huntington's disease and Gilles de la Tourette's syndrome on the ability to hold and shift attention, Neuropsychologia 34(9) (1996), 843-851.

[10] N. Georgiou, J. Bradshaw and J. Phillips, Directed attention in Gilles de la Tourette syndrome, Behavioural Neurology 11 (1998), 85-91.

[11] D.F. Harcherik, J.F. Leckman, J. Detlor and D.J. Cohen, A new instrument for clinical studies of Tourette's syndrome,
Journal of the American Academy of Child Psychiatry 23(2) (1984), 153-160.

[12] E. Kokmen, J.M. Naessens and K.P. Offord, A short test of mental status: Description and preliminary results, Mayo Clinic Proceedings 62 (1987), 281-288.

[13] J. Leckman, B. Peterson, G. Anderson, A. Arnsten, D. Pauls and D. Cohen, Pathogenesis of Tourette's syndrome, Journal Child Psychology and Psychiatry 38(1) (1997), 119-142.

[14] R. Nicoletti, C. Umilta and E. Ladavas, Compatibility due to the coding of the relative position of the effectors, Acta Psychologica 57 (1984), 133-143.

[15] H. Nelson and A. O'Connell, Dementia: The estimation of premorbid intelligence levels using the new adult reading test, Cortex 14(2) (1978), 234-244.

[16] D. Pauls and J. Leckman, The inheritance of Gilles de la Tourette's syndrome and associated behaviors: Evidence for autosomal dominant transmission, New England Journal of Medicine 315(16) (1986), 993-997.

[17] B. Peterson, M.A. Riddel, D.J. Cohen, L.D. Katz, J.C. Smith, M.T. Hardin and J.F. Leckman, Reduced basal ganglia volumes in Tourette's syndrome using three-dimensional reconstruction techniques from magnetic resonance images, $\mathrm{Neu}$ rology 43 (1993), 941-949.

[18] M.I. Posner and C.R. Snyder, Attention and cognitive control, in: Information Processing and Cognition: The Loyola Symposium, R. Solso, ed., Lawrence Erlbaum, Hillsdale, 1975.

[19] M. Posner, J. Walker, F. Friedrich and R. Rafal, Effects of parietal injury on covert orienting of attention, The Journal of Neuroscience 4(7) (1984), 1863-1874.

[20] R.D. Rafal and M.I. Posner, Deficits in human visual spatial attention following thalamic lesions, Proceeding of the National Academy of Sciences of USA 84 (1987), 7349-7353.

[21] R.D. Rafal, M.I. Posner, J.H. Friedman, A.W. Inhoff and E. Bernstein, Orienting attention in Progressive Supranuclear Palsy, Brain 111 (1988), 267-280.

[22] L. Riggio, L.D.G. Gawryszewski and C. Umilta, What is crossed in crossed-hand effects?, Acta Psychologica 62 (1986), 89-100

[23] H.E. Singer, A.L. Reiss, J.E. Brown, E.H. Aylward, B. Shih, E. Chee, E.L. Harris, M.J. Reader, G.A. Chase, R.N. Bryan and M.B. Denckla, Volumetric MRI changes in basal ganglia of children with Tourette's syndrome, Neurology 43 (1993), 950-956.

[24] J. Yesavage and T.L. Brink, Development and validation of a geriatric depression scale: A preliminary report, Journal of Psychiatric Research 17 (1983), 37-49. 


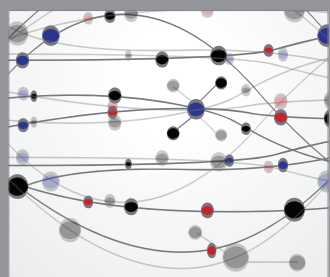

The Scientific World Journal
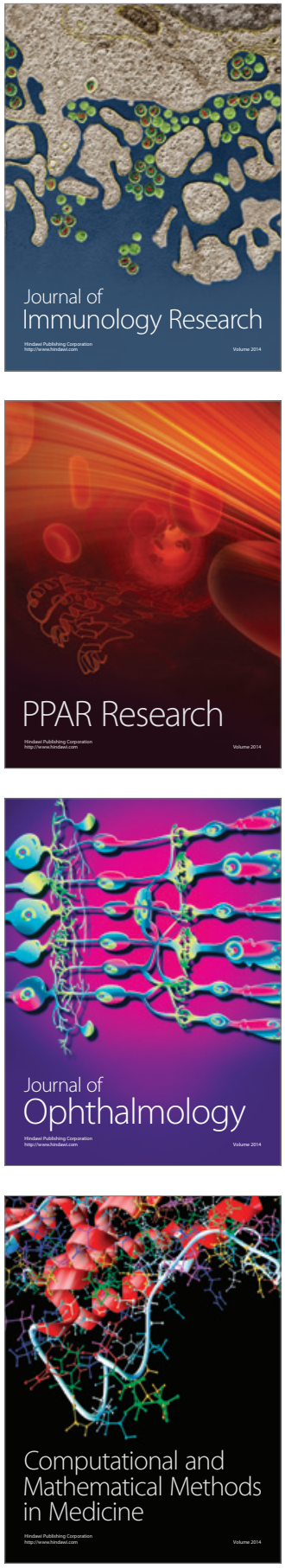

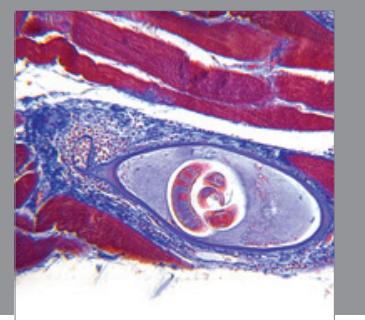

Gastroenterology

Research and Practice
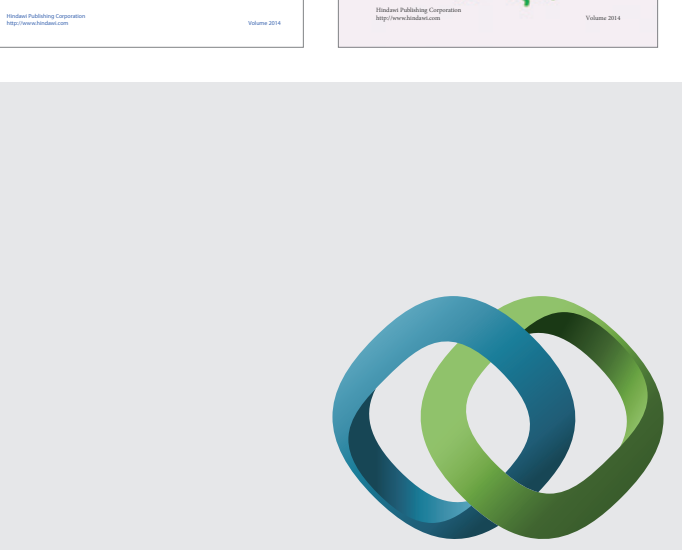

\section{Hindawi}

Submit your manuscripts at

http://www.hindawi.com
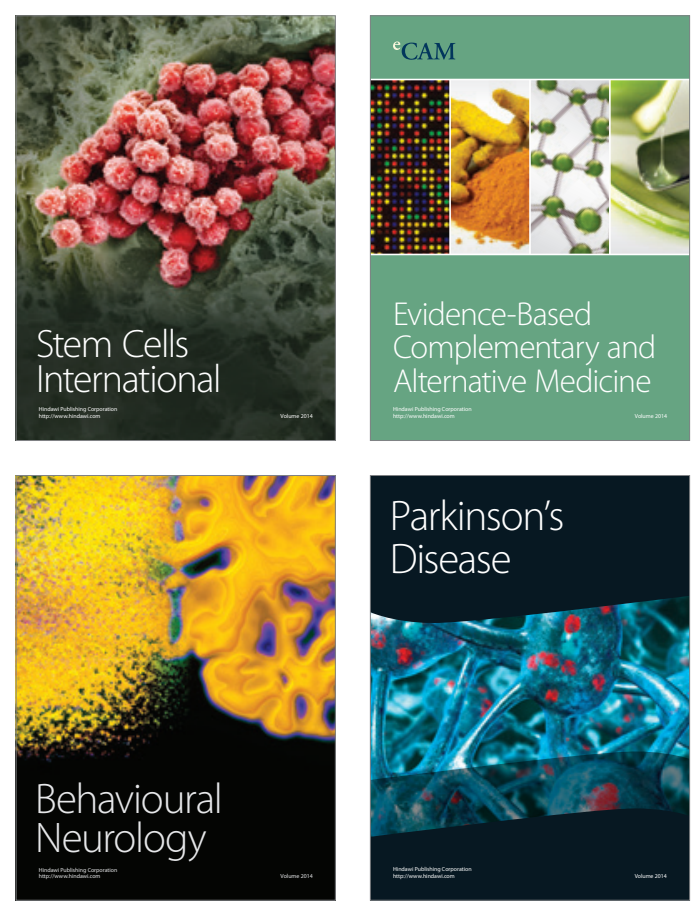

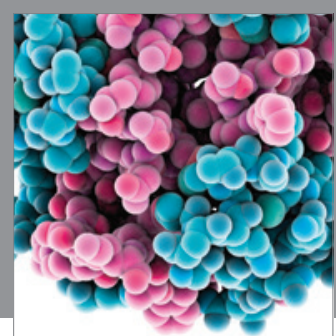

Journal of
Diabetes Research

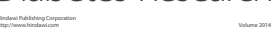

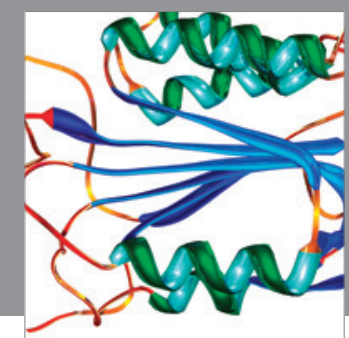

Disease Markers
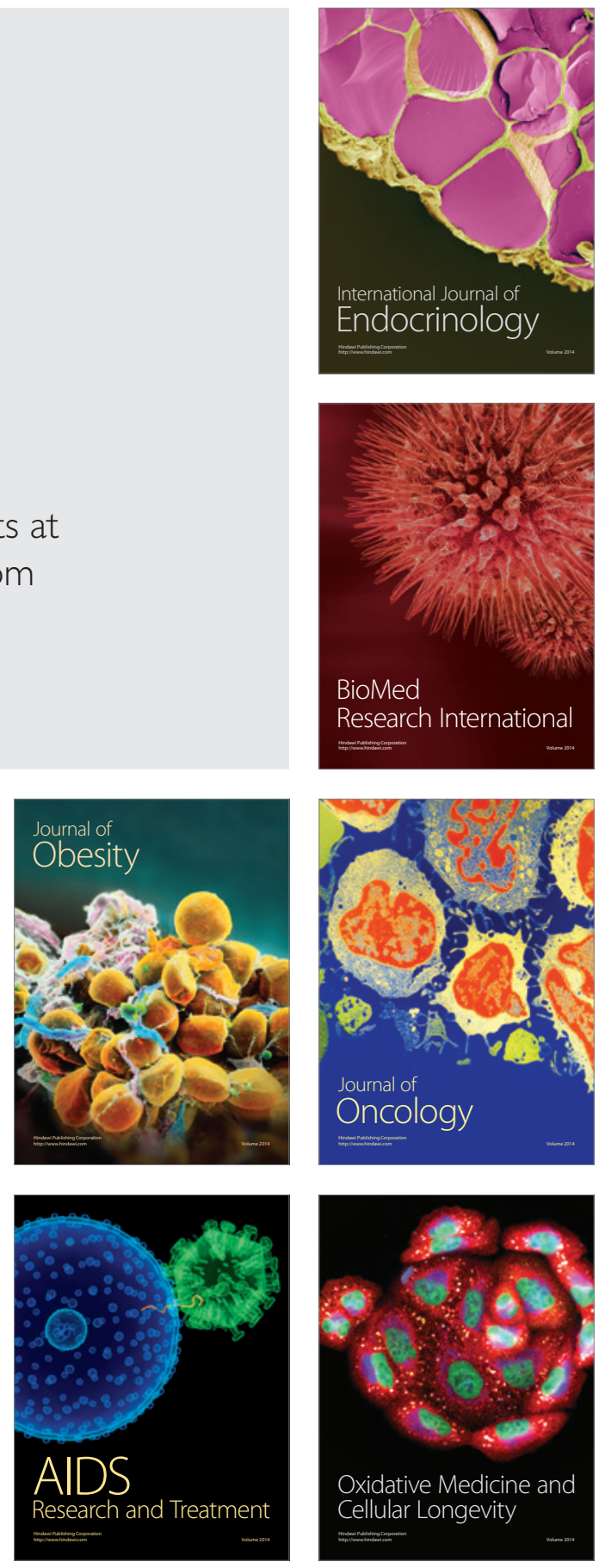